\title{
A Reevaluation of X-Irradiation Induced Phocomelia and Proximodistal Limb Patterning
}

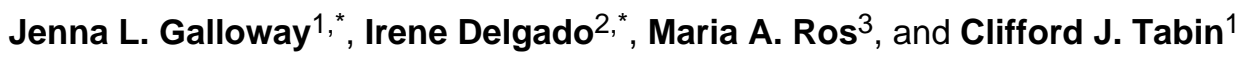 \\ ${ }^{1}$ Department of Genetics Harvard Medical School 77 Avenue Louis Pasteur Boston, \\ Massachusetts 02115, USA \\ ${ }^{2}$ Departamento de Anatomia y Biologia Celular Universidad de Cantabria C/ Herrera Oria s/n \\ 39011 Santander, Espana \\ ${ }^{3}$ Instituto de Biomedicina y Biotecnología de Cantabria (CSIC-UC-IDICAN) C/ Herrera Oria s/n \\ 39011 Santander. Spain
}

\section{Abstract}

Phocomelia is a devastating, rare congenital limb malformation in which the long bones are shorter than normal, with the upper portion of the limb being most severely affected. In extreme cases, the hands or fingers are attached directly to the shoulder and the most proximal elements (those closest to the shoulder) are entirely missing. This disorder, previously known in both autosomal recessive and sporadic forms, showed a dramatic increase in incidence in the early 1960's due to the tragic toxicological effects of the drug thalidomide, which had been prescribed as a mild sedative1, 2. This human birth defect is mimicked in developing chick limb buds exposed to X-irradiation3-5. Both X-irradiation5 and thalidomide-induced phocomelia5, 6 have been interpreted as patterning defects in the context of the Progress Zone Model, which states that a cell's proximodistal (PD) identity is determined by the length of time spent in a distal limb region termed the "Progress Zone" 7. Indeed, studies of X-irradiation induced phocomelia have served as one of the two major experimental lines of evidence supporting the validity of the Progress Zone Model. Here, using a combination of molecular analysis and lineage tracing, we show that X-irradiation-induced phocomelia is fundamentally not a patterning defect, but rather results from a time-dependent loss of skeletal progenitors. As skeletal condensation proceeds from the shoulder to fingers (in a proximal to distal direction), the proximal elements are differentially affected in limb buds exposed to radiation at early stages. This conclusion changes the framework for considering the effect of thalidomide and other forms of phocomelia, suggesting the possibility that the etiology lies not in a defect in the patterning process, but rather in progenitor cell survival and differentiation. Moreover, molecular evidence that PD patterning is unaffected following Xirradiation does not support the predictions of the Progress Zone Model.

According to the Progress Zone Model7, PD structures develop under the influence of a continuous signal, now understood to be a fibroblast growth factor (FGF)8, produced by the

Users may view, print, copy, and download text and data-mine the content in such documents, for the purposes of academic research, subject always to the full Conditions of use:http://www.nature.com/authors/editorial_policies/license.html\#terms

* These authors contributed equally to this work. 
overlying apical ectodermal ridge (AER). Initially, the entire limb mesenchyme has a proximal identity however, the mesenchymal cells at the tip of the limb bud under the AER - those in the "Progress Zone" - are exposed to FGF, allowing them to transition to more distal fates. As the progress zone cells divide and the limb grows, not all of these cells remain within range of the FGF signal. The fate of the cells exiting the progress zone becomes fixed, whereas those in this zone are once again respecified to more distal fates. The Progress Zone Model elegantly explained the effects of X-irradiation on developing limb buds 5 by stating that the X-irradiation-induced cell death and resultantly smaller limb buds cause the proximal cells to remain within range of the AER-produced FGF signal for a longer time than normal, until the limb bud recovers and grows to a sufficient size. Consequently, proximal cells spend an increased amount of time in the progress zone, and ultimately are specified to distal fates5.

The X-irradiation experiments, however, were performed prior to the identification of molecular markers for each limb segment. The use of such markers could provide powerful additional data supporting the Progress Zone-based interpretation of irradiation-induced phocomelia or, alternatively, drawing into question the model's validity. We therefore decided to reexamine this paradigm with modern molecular tools.

We irradiated embryos at embryonic day (E) 3.5 (stage 19-21) and grafted right limb buds onto host wings as described5. Skeletal elements were examined at E9.5 (6 days post irradiation, dpi). As in previous studies 3,4 , the phenotype was dose-dependent. While unirradiated limbs developed normally on the host after grafting (Figure $1 \mathrm{~A}(\mathrm{a}, \mathrm{e})$, minor skeletal malformations such as elbow joint fusion were observed at low doses (Figure $1 \mathrm{~A}(\mathrm{~b}, \mathrm{f}))$. Intermediate doses resulted in the loss of the most proximal element, the humerus, and anterior digits (Figure 1A(c,g)) and at the highest irradiation dose, only digits formed (Figure $1 \mathrm{~A}(\mathrm{~d}, \mathrm{~h})$ ). To eliminate the possibility that the irradiation phenotype could be explained by reabsorption of proximal tissue following grafting, we performed a parallel series of experiments irradiating limbs while shielding the rest of the embryo. Using this method, we generated embryos with shorter limbs lacking proximal elements. Thus, at a dose of 17.5-20 Grays, 28\% irradiated right forelimbs (RFLs) were missing the humerus, and 36\% lacked humerus and radius/ulna ( $\mathrm{n}=36$; Figure 1B(a-c, lower limbs)).

For the Progress Zone Model to explain the phocomelia phenotype, the growth of the limb bud must be impaired by irradiation, while exposure to the permissive AER-FGF signal must not be disrupted. The irradiated limb buds are smaller than unirradiated and contralateral controls at 24, 48 and 72 hours post irradiation (hpi) (Supplementary Figure 2A, C, D). To determine whether FGF signaling continues unabated after X-irradiation, we performed whole mount in situ hybridization, examining Fgf8 expression in irradiated limbs from grafted or shielded embryos. In all irradiated limbs, at all stages examined, we found normal $F g f 8$ expression $(100 \%, n=18$; Supplementary Figure 2A, B). Maintenance of normal AER-FGF signaling levels requires the expression of a second signaling molecule, Sonic hedgehog (Shh), in the posterior limb bud mesenchyme9, 10. Consistent with this, we observed no loss in Shh expression in irradiated limb buds (100\%, n=13; Supplementary Figure 2A, B). Moreover, $A P-2$, a transcription factor dependent on FGF signaling11, was 
expressed in irradiated limbs at all stages analyzed $(100 \%, n=30$; Supplementary Figure 2C, D), verifying that irradiation does not disrupt AER-FGF signaling to limb mesenchyme.

The Progress Zone Model predicts that continual FGF signaling within the geometry of a small limb bud would lead to respecification of proximal tissue to more distal fates, thereby explaining the phocomelia. To verify this hypothesis, we investigated the specification of the three major elements of the limb: the stylopod/humerus, zeugopod/radius-ulna, and autopod/digits. The best markers for these limb segments are, respectively, Meis1/2,

HoxA11, and HoxA13 (Figure 2C). However, their expression patterns are dynamic at early stages and there is scant evidence that these transcription factors are required for PD specification12. Nonetheless, at later stages they uniquely delineate each segment of the limb along the PD axis, providing a molecular indication of their specification. The Progress Zone Model would predict that proximal cells, being respecified to distal fates, would no longer express Meis1,2 and instead express distal markers such as Hoxall or Hoxal3.

Surprisingly, whole mount in situ hybridization (WISH) shows no difference in the relative domains of expression of these markers in irradiated and unirradiated limbs (Figure $2 \mathrm{~A}(\mathrm{q}-\mathrm{s})$ and $2 \mathrm{~B}(\mathrm{i}, \mathrm{j}))$, although at an early time point, expression of Meis1, Meis2, and HoxAll is reduced and HoxA13 expression was virtually undetectable in both grafted and shielded Xirradiated limbs (Figure 2A,B; data not shown). This was likely due to developmental delay as normal forelimb HoxA13 expression initiates after that of HoxA1113. We also found that hindlimb HoxA13 expression was not disrupted following irradiation, consistent with HoxA13 hindlimb expression preceding its expression in the forelimb (data not shown). After 48 hpi, Meis1, Meis2, HoxA11 and HoxA13 expression returned and demarcated the three limb segments. To confirm that there are three distinct PD domains in irradiated limbs, we performed double WISH with Meis1 and HoxA13. Similar to contralateral controls, three distinct domains were identifiable in irradiated limbs, a distal HoxA13 domain, an unstained middle region, and a proximal Meisl domain (Figure 2B(e, f, i, j)).

Our data, thus suggesting that limb segment specification is not affected by $\mathrm{X}$-irradiation, raises the question of why this treatment causes a preferential loss of proximal structures. We reasoned that this could be explained if $\mathrm{X}$-irradiation led to changes in apoptosis, proliferation, or vasculature disproportionately in the proximal limb. X-irradiation causes both apoptosis and cell cycle arrest14. Consistent with this, we see an increase in apoptosis, using TUNEL staining at 3 and 24 hpi (Figure 3A, Supplementary Figure 3 and 4), as well as a $56 \%$ decrease in mitotic cells at 3 hpi by phosphorylated Histone $\mathrm{H} 3(\mathrm{pH} 3)$ staining (Figure 3B, Supplementary Figure 3 and 4). At later stages, neither apoptosis nor proliferation rates differ from contralateral and unirradiated controls or from control and irradiated grafts (Figure 3A(c, f, i); 3B(b, d, f), Supplementary Figure 3). Similar results were reported in prior analyses5. Importantly, however, neither the cell death nor the proliferative changes we observed were localized to or enriched in proximal domains of the limb. Similarly, there do not appear to be any differential changes in the vasculature along the PD axis (data not shown). Thus, these effects would not likely be responsible for the specific failure of the proximal structures to form. 
We next examined whether $\mathrm{X}$-irradiation differentially affects differentiation along the $\mathrm{PD}$ limb axis. In the limb, condensation of the skeletal elements occurs progressively, with proximal elements condensing before distal ones. Sox 9 is an early marker of condensing mesenchyme and is necessary for cartilage and bone formation15. Sox 9 expression is first detected in the limb bud at Stage 21, concurrent with or just after irradiation. We find that at $24 \mathrm{hpi}$, Sox 9 expression is dramatically reduced or lost in irradiated limb buds compared with unirradiated and contralateral controls (Figure 2A(d, l) and 2B(c, d)). After 48 hpi, Sox 9 expression is detected, but, strikingly, only in more distal regions of irradiated limb buds where condensation is initiated at later stages (Figure $2 \mathrm{~A}(\mathrm{~h}, \mathrm{p}, \mathrm{t})$ and $2 \mathrm{~B}(\mathrm{~g}, \mathrm{~h}, \mathrm{k}, \mathrm{l})$ ). Thus, this finding suggests a model in which $\mathrm{X}$-irradiation depletes the number of cells throughout the mesenchyme. This depletion has dire consequences for the proximal tissue, which is in the early stages of chondrogenic condensation, and, as a result of this loss, cannot form skeletal structures. The failure of the proximal cells to condense is likely attributed to insufficient numbers of chondrocyte precursors. More distal tissue, in contrast, has time to recover before the proximal-to-distal wave of differentiation reaches it. A similar model has been proposed to explain segment loss in FGF mutants16, 17. Interestingly, the idea of a threshold number of cells required to form skeletal elements was proposed by Wolpert and colleagues as an explanation as to why structures that have already been specified were affected by increasing doses of irradiation5.

If this model is correct, irradiation at early stages affects the formation of proximal skeletal elements because cells in that domain initiate condensation first, and hence, undergo this process concurrently with the cellular response to irradiation damage. According to this view, we reasoned that irradiation at later stages should selectively affect the later condensing distal elements. We irradiated the limbs of shielded embryos at E4.5 (stage 24) and examined their skeletal elements at E8. The irradiated limbs had severely shortened zeugopods while other limb segments were minimally affected (Figure 4A(arrow in f)). As expected, there was an initial reduction in proliferation and an increase in apoptosis throughout the mesenchyme of the late irradiated limbs (Supplementary Figure 5A; data not shown). Corresponding to the reduction in zeugopod size, Sox 9 expression was abnormal and reduced in the middle segment of irradiated limb specimens despite the presence of HoxA11 expression (Figure 4A(arrow b, e)).

We saw preferentially distal effects when we irradiated later E5.5 (stage 26) limb buds. Most strikingly, a complete loss of the autopod was observed in these irradiated limbs (Figure 4B(arrow in f)). The proximal limb cartilage had less TUNEL positive cells, consistent with our finding that the stylopod and zeugopod are little affected in these limbs and in agreement with the idea that differentiated cartilage is resistant to irradiation-induced cell death 18 (Supplementary Figure 5B). Sox 9 expression was virtually lost in the handplate at 24 and $48 \mathrm{hpi}$, in spite of continued HoxAl3 expression (Figure 4B(b, arrow in e); not shown). Taken together, these results support the idea that $\mathrm{X}$-irradiation at successively later stages sequentially affects more distal limb segments, where chondrogenic condensation is occurring, and as in the earlier stage irradiations, segment specification is not affected.

These conclusions depend on the use of markers which, although congruent with the limb segments, may not be involved in their specification. Therefore, we tested our conclusions in 
a second way. According to the Progress Zone Model where proximal tissue, and indeed, the entire limb bud is respecified as distal following irradiation, all surviving mesenchymal cells of the limb bud, including the proximal ones, should contribute to the distal skeletal structures that form. In contrast, if specification is unaffected, but skeletal elements fail to differentiate from proximal mesenchyme, then proximal tissues should not contribute to the distal elements forming after irradiation.

To follow the fates of proximal tissue after X-irradiation, we injected the lipophilic dye DiI approximately $300 \mu \mathrm{m}$ from the AER in control and irradiated stage 19-20 limb buds. Consistent with the results of previous fate maps19, injection at this location in unirradiated limb buds labels the stylopod segment (Figure 4C(arrowheads in a, d, g)). In contrast, DiI injected proximally in irradiated limbs had limited expansion and did not contribute to the distal skeletal elements that formed (Figure $4 \mathrm{C}$ (arrowheads in b, c, e, f, h, i)). Another lipophilic dye, DiO, however, when injected distally within $50 \mu \mathrm{m}$ of the AER in irradiated limb buds did expand into the distal regions (Supplementary Figure 6). This result supports our hypothesis that the loss of proximal skeletal elements is due to failure of those segment progenitors to condense and not because the cells occupy the progress zone for longer periods and are respecified to distal fates.

Our data, in conjunction with earlier studies reevaluating the effect of AER removal20, eliminate the major experimental support for the Progress Zone Model. While our study does not address the mechanism by which PD specification does occur, it is consistent with models in which specification is driven by the influence of traditional signaling centers12, 21 rather than by time spent within a progress zone. Most importantly, our data indicate that phocomelia caused by $\mathrm{X}$-irradiation results from a loss of prechondrogenic progenitors and a consequent failure in differentiation, rather than from a defect in PD patterning.

Increased cell death has been thought to underlie thalidomide-induced limb truncations in chick embryos, but whether this is a result of direct activation of caspase pathways22, 23, or an indirect result of angiogenic inhibition, remains unclear 24. Although thalidomide treatment in chick causes distal truncations, thalidomide's effects in humans on predominantly proximal segments likewise have been suggested to be due to cell death. Indeed, cell death has been linked to phocomelia in other contexts of teratogenic exposure, such as with nitrogen mustard25, 26 and the phocomelia observed in these experiments has been interpreted mainly as a patterning defect5,6. However, our results suggest that in these cases, as in the irradiation experiments, cell death may lead to phocomelia not by producing a smaller limb bud in the context of a Progress Zone, but by eliminating chondrogenic precursors during a time window when proximal condensation is compromised but distal differentiation has not yet commenced.

\section{Supplementary Material}

Refer to Web version on PubMed Central for supplementary material. 


\section{Acknowledgements}

We would like to thank José Gómez Arozamena (Física Médica, Universidad de Cantabria) and David Connor (Genetics Department, HMS) for help with the irradiation machine and members of the Tabin-Cepko and Ros laboratories for helpful discussions and ideas. Work in the laboratory of C.J.T is supported by a grant, R37 HD032443, from the NIH. J.L.G. is supported by grant F32HD057701 from the National Institute of Child Health and Human Development. I.D. and M.R are supported by BFU2008-00397 from the Spanish Ministry of Science and Innovation.

\section{Methods Summary}

Eggs were obtained, maintained and incubated as described20. In ovo irradiations were performed at the Hamburger Hamilton stages indicated. For the grafted limb experiments, unirradiated and irradiated stage 19-21 limb buds were dissected and grafted to the anterior wing border of a stage 24-25 host embryo as described5 to overcome the subsequent death of the embryo. In ovo irradiations were performed with eggshell shielding with a 2-3 mm diameter hole, exposing the RFL. This protected the embryo and the blood islands from damage while the RFL was irradiated.

Whole mount in situ hybridizations and Alcian Blue and Alizarin Red skeletal preparations were performed as described27, 28. Chick probes were Meis1 and Meis221, HoxA11 and HoxA1313, Fgf8 (gift of J. C. Izpisua Belmonte), Shh29, and Sox9 30. AP-2 (ChEST765g1) was linearized with Not I and antisense probe was generated using T3. For each gene expression, irradiation dose and time period, we have analyzed at least two irradiated grafted specimens (both irradiated leg and wing grafts were used) and at least three irradiated limbs from shielded embryos. In situ Cell Death Detection Kit, TMP red (Roche) and ApoTag Fluorescein in situ Detection Kit (Chemicon Int.) were used for TUNEL. pH3 immunohistochemistry was performed with a-pH3 (1:200, Millipore) and anti-rabbit AlexaFluor 594 (1:200, Invitrogen) or biotinylated secondary antibody. All images of irradiated RFLs and LFLs are at the same magnification.

The lipophilic dyes DiI and/or DiO (Invitrogen) were prepared and injected into limb buds 1-2 hours after irradiation as described 19,20. After photographing, limbs were stained to visualize the cartilage and skeletal elements and were compared with the previous fluorescent images at the same magnification.

The online version of the paper contains a more detailed description of the methods described here.

\section{Methods \\ Irradiation}

For the grafted limb experiments, a Xylon International Smart 200-E irradiator at 200kV was used at a dose rate of $2.2 \mathrm{~Gy} / \mathrm{min}$ at a height of $18 \mathrm{~cm}$. Three different doses were used (low, 3.6; intermediate, 5.5; high,7.25 Gy) that were selected according to the resulting phenotypes. For the in ovo irradiations, the RFL bud was exposed to 17.5 to $20 \mathrm{~Gy}$ (1750-2000 rads) in a Faxitron 43855D irradiator at $120 \mathrm{kV}$ and a height of $30.5 \mathrm{~cm}$. This dose range consistently produced phocomelia in RFLs and never in LFLs. Some irradiated 
specimens lack anterior (Figure 1B(a, b; lower limbs)) and posterior digits (Figure 1B(c; lower limb)). On a few occasions, the contralateral LFLs had mild phenotypes, including abnormally shaped skeletal elements (Figure 1B, asterisk; humerus in upper limb of a) or fusion at the elbow (Figure 1B, asterisk; upper limb in c), but never lost proximal elements. While the phenotypes produced by shielding were more variable than the grafting method (likely due to variation in shield placement), we consistently saw phocomelia phenotypes in the affected limbs. Irradiated limbs from shielded embryos were only collected and used for in situ hybridizations, TUNEL or $\mathrm{pH} 3$ analysis if they were morphologically smaller and appeared affected compared with the contralateral LFLs.

\section{Whole mount in situ hybridizations and skeletal preparations}

After overnight fixation in 4\% paraformaldehyde/PBS and subsequent dehydration in methanol, in situ hybridizations were performed as described 27. DIG-labeled probes were detected with NBT/BCIP (Sigma) and FITC-labeled probes were detected with INT/BCIP (Sigma). Cartilage and bone staining were performed after harvesting at E7-9.5 using Alcian Blue and Alizarin Red as described28.

\section{Analysis of cell death, proliferation and the vasculature}

Limbs were fixed in 4\% PFA for 1-2 hours, dehydrated in ethanol, cleared in xylene, embedded in paraffin, and 7-10 $\mu \mathrm{m}$ alternate sections were collected and used for TUNEL staining and pH3 immunohistochemistry. TUNEL staining was done using the In situ Cell Death Detection Kit, TMR red (Roche) or ApoTag Fluorescein in Situ Detection Kit (Chemicon Int.). For pH3-staining, antigen retrieval was followed sequentially by incubation with a-pH3 (1:200, Millipore) and anti-rabbit AlexaFluor 594 (1:200, Invitrogen) or biotinylated secondary antibody followed by incubation with ABC solution (Vector Laboratories) and development in diaminobenzidine. Slides for TUNEL and a-pH3 were counterstained with DAPI to visualize the nucleus. In some irradiated-shielded specimens, there were more apoptotic cells on the dorsal side closest to irradiation exposure (upper portion of limb in Figure 3A(e)). The number of TUNEL and pH3 positive cells were counted in irradiated RFLs, contralateral LFLs, and unirradiated limbs from shielded embryos at $3 \mathrm{hpi}$ and this number was divided by the total pixel area of the limb for Supplementary Figure 4. When calculating the pixel area, all images were at the same magnification and resolution. For both sets of data, 2 sections from at least 4 different irradiated specimens were counted.

Using an Eppendorf pressure injector (Model 5242), approximately $3 \mu$ of Alexa Fluor ${ }^{\circledR}$ 488 Acetylated LDL (Invitrogen) was directly injected into the circulation of control and irradiated embryos. AcLDL binds and is internalized by endothelial cells and macrophages and it was used to visualize changes in the vasculature at several timepoints after irradiation.

\section{Lineage tracing}

The lipophilic dyes DiI and/or DiO (Invitrogen) were injected into stage19-20 limb buds 1-2 hours after irradiation as described using a calibrated reticle19, 20. Embryos were allowed to develop for 4-5 days before harvesting and imaging for fluorescent signals on a Nikon 
Eclipse E1000 with Y-2 E/c Texas Red (DiI) and fluorescein isothiocyanate/HYQ (DiO) filters.

\section{References}

1. Lenz W. Thalidomide and congenital abnormalities. Lancet. 1962; 1:45.

2. Toms D. Thalidomide and congenital limb abnormalities. Lancet. 1962; 2:400.

3. Goff RA. The relation of development status of limb formation of x-radiation sensitivity in chick embryos. I. Groso study. J Exp Zool. 1962; 151:177-200. [PubMed: 13963311]

4. Pinot M. Developpement de l'ebauche des Membres apres traitement a l'yperite azotee, irradiation aux rayons X et culture in vitro. Etude comparative chez l'embryon de poulet. Annal. Embryol. Morphog. 1970; 3:215-234.

5. Wolpert L, Tickle C, Sampford M. The effect of cell killing by $x$-irradiation on pattern formation in the chick limb. J Embryol Exp Morphol. 1979; 50:175-93. [PubMed: 458354]

6. Tabin CJ. A developmental model for thalidomide defects. Nature. 1998; 396:322-3. [PubMed: 9845068]

7. Summerbell D, Lewis JH, Wolpert L. Positional information in chick limb morphogenesis. Nature. 1973; 244:492-6. [PubMed: 4621272]

8. Niswander L, Tickle C, Vogel A, Booth I, Martin GR. FGF-4 replaces the apical ectodermal ridge and directs outgrowth and patterning of the limb. Cell. 1993; 75:579-87. [PubMed: 8221896]

9. Niswander L, Jeffrey S, Martin GR, Tickle C. A positive feedback loop coordinates growth and patterning in the vertebrate limb. Nature. 1994; 371:609-12. [PubMed: 7935794]

10. Laufer E, Nelson CE, Johnson RL, Morgan BA, Tabin C. Sonic hedgehog and Fgf-4 act through a signaling cascade and feedback loop to integrate growth and patterning of the developing limb bud. Cell. 1994; 79:993-1003. [PubMed: 8001146]

11. Shen $\mathrm{H}$, et al. Chicken transcription factor AP-2: cloning, expression and its role in outgrowth of facial prominences and limb buds. Dev Biol. 1997; 188:248-66. [PubMed: 9268573]

12. Tabin C, Wolpert L. Rethinking the proximodistal axis of the vertebrate limb in the molecular era. Genes Dev. 2007; 21:1433-42. [PubMed: 17575045]

13. Nelson CE, et al. Analysis of Hox gene expression in the chick limb bud. Development. 1996; 122:1449-66. [PubMed: 8625833]

14. Choudhury A, Cuddihy A, Bristow RG. Radiation and new molecular agents part I: targeting ATM-ATR checkpoints, DNA repair, and the proteasome. Semin Radiat Oncol. 2006; 16:51-8. [PubMed: 16378907]

15. Akiyama H, Chaboissier MC, Martin JF, Schedl A, de Crombrugghe B. The transcription factor Sox9 has essential roles in successive steps of the chondrocyte differentiation pathway and is required for expression of Sox5 and Sox6. Genes Dev. 2002; 16:2813-28. [PubMed: 12414734]

16. Sun X, Mariani FV, Martin GR. Functions of FGF signalling from the apical ectodermal ridge in limb development. Nature. 2002; 418:501-8. [PubMed: 12152071]

17. Mariani FV, Martin GR. Deciphering skeletal patterning: clues from the limb. Nature. 2003; 423:319-25. [PubMed: 12748649]

18. Biggers JD, Gwatkin RB. Effect of X-Rays on the Morphogenesis of the Embryonic Chick Tibiotarsus. Nature. 1964; 202:152-4. [PubMed: 14156290]

19. Sato K, Koizumi Y, Takahashi M, Kuroiwa A, Tamura K. Specification of cell fate along the proximal-distal axis in the developing chick limb bud. Development. 2007; 134:1397-406. [PubMed: 17329359]

20. Dudley AT, Ros MA, Tabin CJ. A re-examination of proximodistal patterning during vertebrate limb development. Nature. 2002; 418:539-44. [PubMed: 12152081]

21. Mercader N, et al. Opposing RA and FGF signals control proximodistal vertebrate limb development through regulation of Meis genes. Development. 2000; 127:3961-70. [PubMed: 10952894]

22. Knobloch J, Shaughnessy JD Jr. Ruther U. Thalidomide induces limb deformities by perturbing the Bmp/Dkk1/Wnt signaling pathway. Faseb J. 2007; 21:1410-21. [PubMed: 17283219] 
23. Knobloch J, Schmitz I, Gotz K, Schulze-Osthoff K, Ruther U. Thalidomide induces limb anomalies by PTEN stabilization, Akt suppression, and stimulation of caspase-dependent cell death. Mol Cell Biol. 2008; 28:529-38. [PubMed: 18178729]

24. Therapontos C, Erskine L, Gardner E, Figg W, Vargesson N. Thalidomide induces limb defects by preventing angiogenic outgrowth during early limb formation. Proc Natl Acad Sci U S A. 2009 in press.

25. Salzgeber B. Comparative study of the effects of nitrogen mustard on mesodermal and ectodermal limb bud components of chick embryos. J Embryol Exp Morphol. 1969; 22:373-94. [PubMed: 5360023]

26. Salzgeber B. Ectodermal-mesodermal interactions in chick embryo limb buds treated with nitrogen mustard. Resumes au Congres Franco-Japonais. 1975; 17:295-6.

27. Wilkinson DG, Nieto MA. Detection of messenger RNA by in situ hybridization to tissue sections and whole mounts. Methods Enzymol. 1993; 225:361-73. [PubMed: 8231863]

28. McLeod MJ. Differential staining of cartilage and bone in whole mouse fetuses by alcian blue and alizarin red S. Teratology. 1980; 22:299-301. [PubMed: 6165088]

29. Riddle RD, Johnson RL, Laufer E, Tabin C. Sonic hedgehog mediates the polarizing activity of the ZPA. Cell. 1993; 75:1401-16. [PubMed: 8269518]

30. Healy C, Uwanogho D, Sharpe PT. Regulation and role of Sox9 in cartilage formation. Dev Dyn. 1999; 215:69-78. [PubMed: 10340758] 

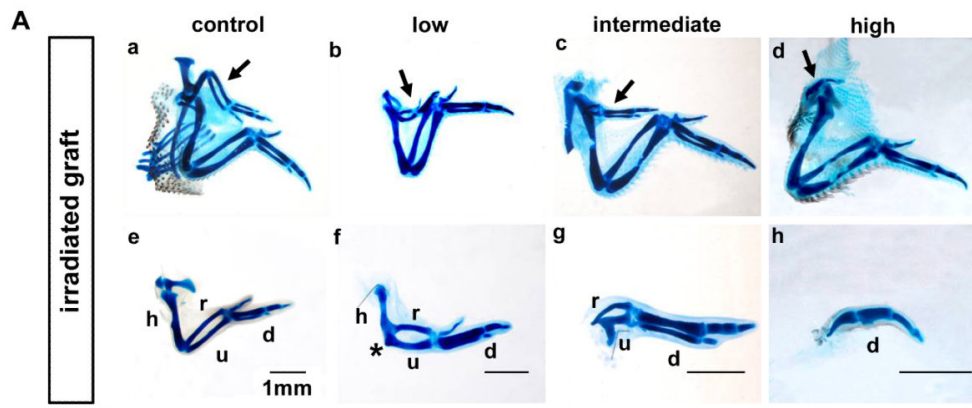

B

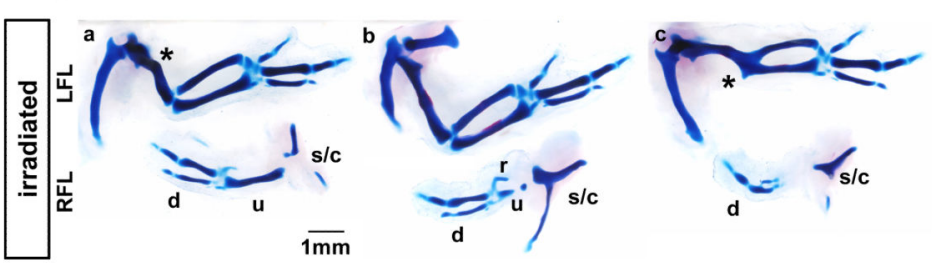

Figure 1.

$\mathrm{X}$-irradiation of chick limbs causes phocomelia.

(A) Limb grafts (a - d, arrows; e-h) are shown next to host limbs. Controls (a,e) develop a humerus (h), radius(r), ulna (u), and digits (d). Low doses cause elbow fusion (asterisk) and reduced anterior digit 2 (f). Intermediate dosed-limbs have no humerus, but form a partial radius and ulna and normal posterior digits (c, g). At high doses, only digits form (d,h).

(B) Irradiated RFLs from shielded embryos (lower limb; a-c) lose proximal elements compared to LFLs (upper limb; a-c). Some irradiated specimens lack an anterior (a, b) or posterior digit (c, lower limb). Occasionally, LFLs had mild abnormalities (asterisks). a-c are ventral images at the same scale. Scale bars $=1 \mathrm{~mm}$ 


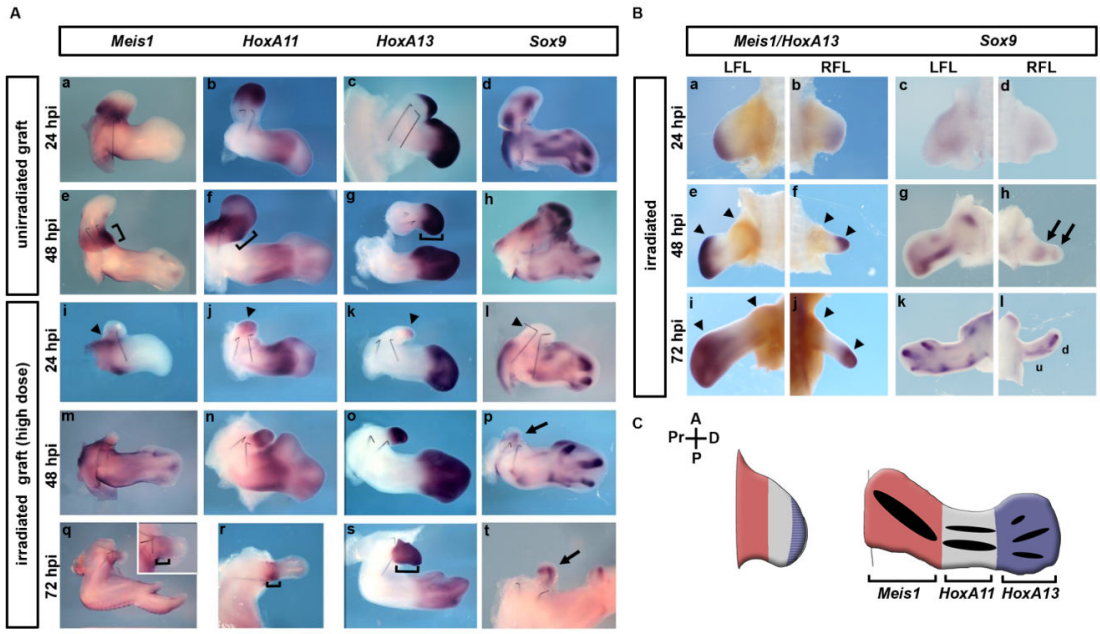

Figure 2.

Expression analysis of irradiated limbs reveals defects in differentiation but not specification of the proximal segments.

(A) In irradiated limb grafts, Meis1, HoxA11, and HoxA13 expression is reduced at 24 hpi (i, $\mathrm{j}, \mathrm{k}, \mathrm{l}$, arrowheads) compared with controls (a-d). At later timepoints, expression demarcates three limb segments (m-o; q-s, brackets) as in controls (e-g, brackets). Sox 9 expression is found distally (p, t, arrows) compared with controls $(\mathrm{d}, \mathrm{h})$. (Grafted limbs are located in the anterior margin of host limbs.)

(B) At 24 hpi, irradiated RFLs displayed reduced expression of Meisl (brown), HoxA13 (purple; b; 91\%, n=11) and Sox9 (d; 100\%, n=6) compared with LFLs (a, c). After 48 hpi, Meis 1 and HoxA13 expression in irradiated RFLs (f, $\mathrm{j}$, arrowheads; 100\%, $\mathrm{n}=12$ ) was similar to LFLs (e, i, arrowheads). Sox9 was only expressed distally in irradiated RFLs (100\%, n=9) at $48 \mathrm{hpi}$ (h, arrows). $\mathrm{k}$ and 1 were stained at 96hpi (u, ulna; d, digit).

(C) At stage 23 (left), Meis1 (red) is proximal; HoxA11 (grey) is distal and partially overlapping with HoxA13 (purple). After stage 28 (right), expression demarcates three segments (Pr, proximal; D, distal; A, anterior; P, posterior). 


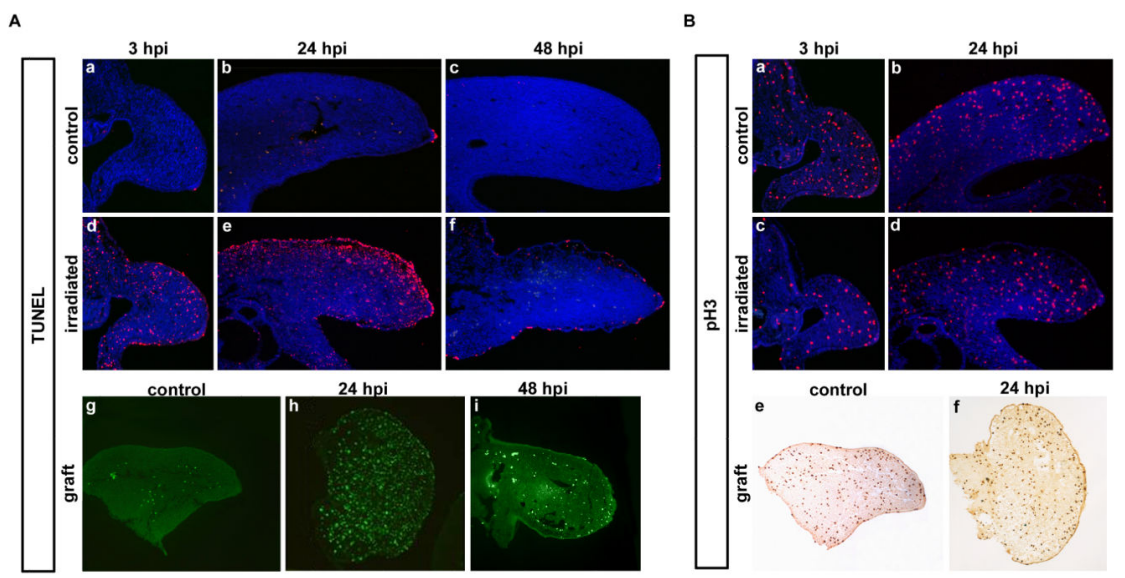

Figure 3.

Changes in cell death and proliferation do not explain the loss of proximal structures.

(A) An increase in TUNEL-positive cells in irradiated RFLs from shielded embryos (d, e) and irradiated grafts (h) compared with unirradiated controls ( $\mathrm{a}, \mathrm{b}, \mathrm{g})$. Levels are relatively normal at 48 hpi (c, f, i). Autofluorescence in ( $g$, i) is due to blood cells.

(B) A decrease in pH3-positive cells at $3 \mathrm{hpi}$ in irradiated RFLs (c) compared to controls (a). By 24 hpi, pH3-staining of irradiated RFLs (d) and grafts (f) is similar to controls (c, e). Sections are longitudinal except for $\mathrm{h}$ and $\mathrm{f}$, which are frontal. 


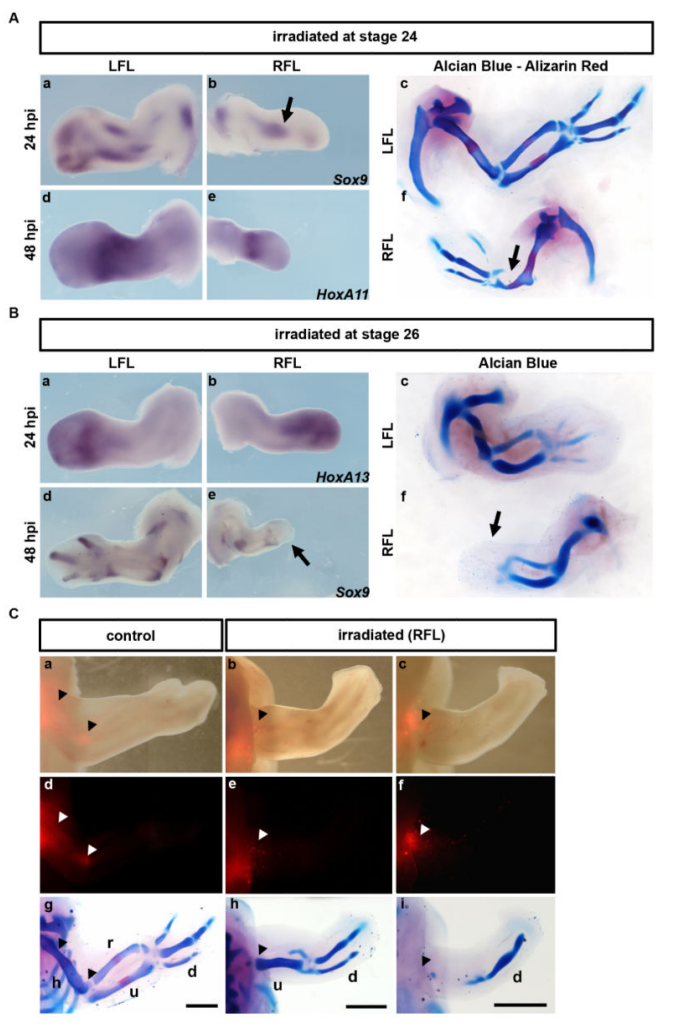

Figure 4.

$\mathrm{X}$-irradiation disrupts chondrogenesis and does not convert proximal cells to distal fates.

(A) Expression of $\operatorname{Sox} 9(\mathrm{~b} ; 100 \%, \mathrm{n}=8)$ and HoxA11 (100\%, $\mathrm{n}=6)$, and skeletal preparations of stage 24 irradiated RFLs (b, e, f; arrows) and LFLs (a, c, d).

(B) Expression of HoxA13 (100\%, n=3) and Sox9 (100\%, n=3) and Alcian Blue-staining of stage 26 irradiated RFLs (b, e, f; arrows) and LFLs (a, c, d).

(C) DiI (red fluorescence) labels the humerus in controls, (a, d, g, arrowheads; 100\%, n=5). In irradiated RFLs, DiI did not expand distally (b, e, h, arrowheads; 100\%, n=4 and c, f, i, arrowheads; $100 \%, \mathrm{n}=2$ ). Scale bar $=1 \mathrm{~mm}$; $\mathrm{h}$, humerus; $\mathrm{r}$, radius; $\mathrm{u}$, ulna; d, digits. 